\title{
MicroRNA-21-5p targeting PDCD4 suppresses apoptosis via regulating the PI3K/AKT/FOXO1 signaling pathway in tongue squamous cell carcinoma
}

\author{
CHANGFU LIU $^{1 *}$, ZHOU TONG $^{1 *}$, JINGYU TAN $^{2}$, ZENGXI XIN $^{3}$, ZHIYING WANG $^{4}$ and LUMING TIAN ${ }^{1}$ \\ ${ }^{1}$ Department of Oral and Maxillofacial Surgery, The Second Affiliated Hospital of Jinzhou Medical University; \\ ${ }^{2}$ Department of Stomatology, The First Affiliated Hospital of Jinzhou Medical University; \\ Departments of ${ }^{3}$ Prosthodontics and ${ }^{4}$ Dental Implants, The Second Affiliated Hospital of Jinzhou \\ Medical University, Jinzhou, Liaoning 121000, P.R. China
}

Received February 25, 2019; Accepted July 29, 2019

DOI: $10.3892 /$ etm.2019.7970

\begin{abstract}
The aim of the present study was to analyze the role of microRNA (miRNA)-21-5p in tongue squamous cell carcinoma (TSCC), predict the target gene of miR-21-5p and provide novel strategies for gene therapy in TSCC treatment. The expression levels of miRNA-21-5p in TSCC tissues were analyzed using reverse transcription quantitative polymerase chain reaction, and the effects of miRNA-21-5p on cell proliferation, invasion and apoptosis and the expression levels of target protein PDCD4 in the $\mathrm{Cal} 27$ and SCC 9 cell lines were determined.PI3K/AKT/Forkhead Box O1 (FOXO1) pathway-associated protein expression levels were evaluated by western blot analysis. miRNA-21-5p was consistently upregulated in TSCC tissues compared with normal tissues. Inhibition of miR-21-5p inhibited cell proliferation and invasion, and promoted cell apoptosis. A luciferase reporter assay confirmed that PDCD4 was the target of miR-21-5p. Inhibition of miRNA21-5p suppressed the PI3K/Akt/FOXO1 signaling pathway. The results from the present study indicated that miR-21-5p-targeting PDCD4 suppresses apoptosis in human
\end{abstract}

Correspondence to: Dr Luming Tian, Department of Oral and Maxillofacial Surgery, The Second Affiliated Hospital of Jinzhou Medical University, Section 2, 49 Shanghai Road, Guta, Jinzhou, Liaoning 121000, P.R. China

E-mail: memopoor@163.com

Dr Zhiying Wang, Department of Dental Implants, The Second Affiliated Hospital of Jinzhou Medical University, Section 2, 49 Shanghai Road, Guta, Jinzhou, Liaoning 121000, P.R. China

E-mail: bdwzy@126.com

*Contributed equally

Abbreviations: TSCC, tongue squamous cell carcinoma; miRNA, microRNA; PDCD4, programmed cell death 4; WT, wild-type

Key words: microRNA-21-5p, tongue squamous cell carcinoma, apoptosis, programmed cell death 4, phosphoinositide 3-kinase pathway
TSCC cell lines. This anti-apoptotic effect was achieved by regulating the PI3K/Akt/FOXO1 signaling pathway. These data represent the basis for a promising novel strategy for the treatment of TSCC.

\section{Introduction}

Tongue squamous cell carcinoma (TSCC) is the most common type of oral malignancy, accounting for $40-50 \%$ of all cases (1). TSCC exhibits the characteristics of high malignancy, rapid growth and marked levels of infiltration; furthermore, the tongue has abundant lymphatic vessels and blood circulation within its structure, which often results in TSCC progression to lymph node metastasis at earlier stages, and it seriously affects the voice, chewing and swallowing ability of the patients (2-4). At present, treatment of TSCC primarily relies on surgery, and treatment via radiotherapy and chemotherapy is auxiliary. However, the 5-year survival rate of patients is only $32-54 \%$ following comprehensive treatment $(5,6)$. Therefore, identifying effective treatment methods is essential to improve survival rates for these patients.

Current research regarding TSCC focuses on microRNAs (miRNAs), and the function of miRNAs has preliminarily been identified. miRNA are small non-coding RNA molecules measuring 18-23 nucleotides in length. Although miRNAs have a small molecular weight, they serve a role in the transcriptional level of human cells and regulates numerous important biological functions of both plant and animal organisms, and in human tumors $(7,8)$.

mi-21-5p is one of the most important members of the miRNA family, and it is closely associated with the occurrence and development of cancer. miRNA-21-5p, located on chromosome 17q23.1, has been identified in numerous different types of tumors, which indicates that it may exhibit enhanced expression levels that correspond with disease development. Jiang et al (9) revealed that miRNA-21-5p was upregulated in gastric cancer tissues and SGC-7901 cells, and that the knockdown of miRNA-21-5p suppressed cell proliferation, migration and invasion, and the inflammatory response. The identification of miRNAs and their expression profiles among 
different diseases indicates that miRNA-21 may serve as a potential biomarker (9). However, the role of miR-21-5p in TSCC and its associated underlying molecular mechanisms have not yet been reported.

In the present study, the expression levels of miR-21-5p in TSCC were investigated, and the apoptotic effect of miRNA-21-5p on human TSCC Cal 27 and SCC9 cell lines was examined. The results revealed that the PI3K/AKT signaling pathway serves a role in the underlying molecular mechanism of the disease.

\section{Materials and methods}

Patients and tissue samples. In total, 40 tumor tissue samples were obtained from patients with TSCC who had been admitted to the Department of Oral and Maxillofacial Surgery, Second Affiliated Hospital of Jinzhou Medical University (Jinzhou, China) between January 2017 and June 2018, including 24 males and 16 females, aged 38-76 years, with a median age of 54 years. None of the patients received chemotherapy or radiotherapy. In addition, 40 cases of normal tissues (adjacent non-cancerous tissues) were obtained from the Second Affiliated Hospital of Jinzhou Medical University. The inclusion criteria were as follows: All patients were diagnosed with TSCC via pathology, and no radiation therapy or chemotherapy was performed prior to biopsy. The exclusion criteria were as follows: Patients with one or more of the following conditions were excluded: i) Infectious disease; ii) acute cardiovascular and cerebrovascular diseases; iii) rheumatic disease; iv) diabetes; or v) other tumors.

The present study was approved by the Ethical Committee of Jinzhou Medical University on October 26, 2016 (approval no. JZH2016052). Written informed consent was obtained from all patients included in the present study.

Hematoxylin and eosin $(H \& E)$ staining. TSCC tissues were fixed ( $>24 \mathrm{~h}$ at room temperature) in $4 \%$ paraformaldehyde and embedded in paraffin. Paraffin-embedded samples were then sliced into $4 \mu \mathrm{m}$ sections and resected specimens were dewaxed in xylene, washed in distilled water and stained with hematoxylin and eosin at room temperature for $5 \mathrm{~min}$. Pathological alterations of myocardial tissue were observed under a light microscope (magnification, x200).

Cell culture. TSCC Cal 27 and SCC9 cell lines were purchased from The Cell Bank of Type Culture Collection of Chinese Academy of Sciences. The Cal 27 cell line was cultured with Dulbecco's modified Eagle's medium (DMEM; Gibco; Thermo Fisher Scientific, Inc.) containing 10\% fetal bovine serum (FBS; Gibco; Thermo Fisher Scientific, Inc.) in a 5\% $\mathrm{CO}_{2}$ incubator at $37^{\circ} \mathrm{C}$ and saturated humidity. The SCC9 cell line was incubated with RPMI-1640 (Gibco; Thermo Fisher Scientific, Inc.) containing $10 \% \mathrm{FBS}$ in a $5 \% \mathrm{CO}_{2}$ incubator at $37^{\circ} \mathrm{C}$.

Reverse transcription-quantitative polymerase chain reaction ( $R T-q P C R)$. Total RNA was extracted from the tissues or cell lines using TRIzol ${ }^{\circledR}$ reagent according to manufacturer's protocol. The cDNA was transcribed using
Table I. Characteristic features of study subjects.

\begin{tabular}{|c|c|c|}
\hline Characteristics & $\begin{array}{c}\text { Patients } \\
\text { with TSCC }\end{array}$ & $\begin{array}{l}\text { Non-cancerous } \\
\text { tissues }\end{array}$ \\
\hline \multicolumn{3}{|l|}{ Age, years } \\
\hline Range & $38-76$ & $35-71$ \\
\hline Mean \pm SD & $57 \pm 9.25$ & $59 \pm 11.38$ \\
\hline Smoking & 21 & 20 \\
\hline Non-smoking & 19 & 20 \\
\hline Drinking & 15 & 20 \\
\hline Non-drinking & 25 & 20 \\
\hline Local stimulation & 5 & - \\
\hline $\begin{array}{l}\text { Residual roots and } \\
\text { crowns of teeth }\end{array}$ & 3 & \\
\hline Bad prosthesis & 2 & \\
\hline Tumor location & & - \\
\hline Lingual margin & 26 & \\
\hline Lingual root & 8 & \\
\hline Ventral of tongue & 6 & \\
\hline Tumor size & & - \\
\hline $\mathrm{T} 1$ & 4 & \\
\hline $\mathrm{T} 2$ & 21 & \\
\hline T3 & 10 & \\
\hline $\mathrm{T} 4$ & 5 & \\
\hline Lymph node involvement & & - \\
\hline No & 23 & \\
\hline $\mathrm{N}+$ & 17 & \\
\hline Pathological classification & & - \\
\hline Squamous cell carcinoma & 40 & \\
\hline Histological classification & & - \\
\hline Well differentiated & 11 & \\
\hline Moderately differentiated & 24 & \\
\hline Poorly differentiated & 5 & \\
\hline Clinical stage & & - \\
\hline I & 4 & \\
\hline II & 12 & \\
\hline III & 17 & \\
\hline IV & 7 & \\
\hline
\end{tabular}

SD, standard deviation; TSCC, tongue squamous cell carcinoma.

a Prime Script ${ }^{\mathrm{TM}}$ RT Master Mixture according to the manufacturer's protocol (Takara Biotechnology Co., Ltd.). miR-21-5p in TSCC was detected using SYBR Prime Script miRNA RT-PCR kit (Takara Biotechnology Co., Ltd.). The thermocycling conditions were as follows: Pre-denaturation at $95^{\circ} \mathrm{C}$ for $1 \mathrm{~min}$, followed by denaturation at $95^{\circ} \mathrm{C}$ for $15 \mathrm{sec}$, annealing at $60^{\circ} \mathrm{C}$ for $40 \mathrm{sec}$ and extension at $72^{\circ} \mathrm{C}$ for $15 \mathrm{sec}$, for a total of 40 cycles. The primer sequences in the present study were as follows: hsa-miR-21-5p forward, 5'-GGGGTA GCTTATCAGACTGATG-3'; hsa-miR-21-5p reverse, 5'-TGT CGTGGAGCGGCAATTG-3'; U6: Forward, 5'-CGCTTC GGCACATATACTA-3'; U6 reverse, 5'-CGCTTCACGAAT TTGCGTGTCA-3'; PDCD4 forward, 5'-TGTGCCAACCAG 


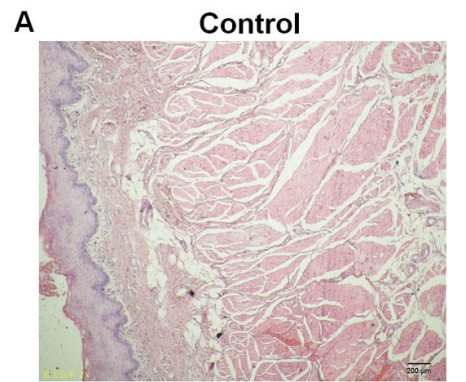

C

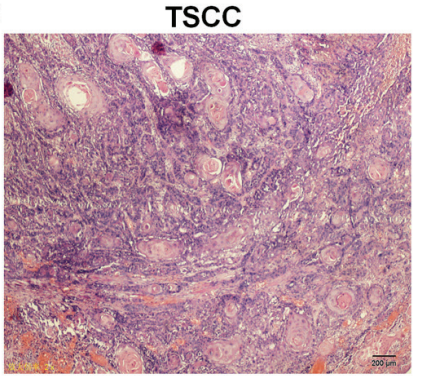

B

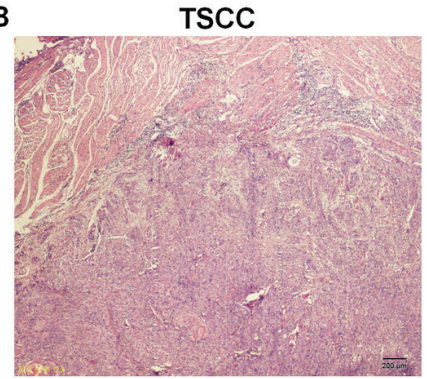

D

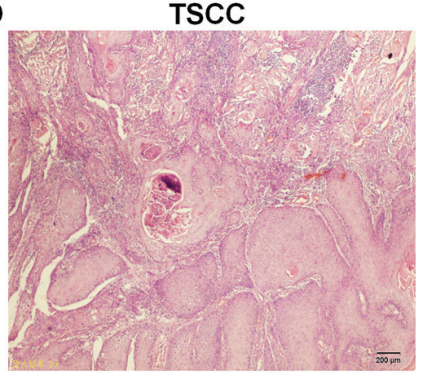

E

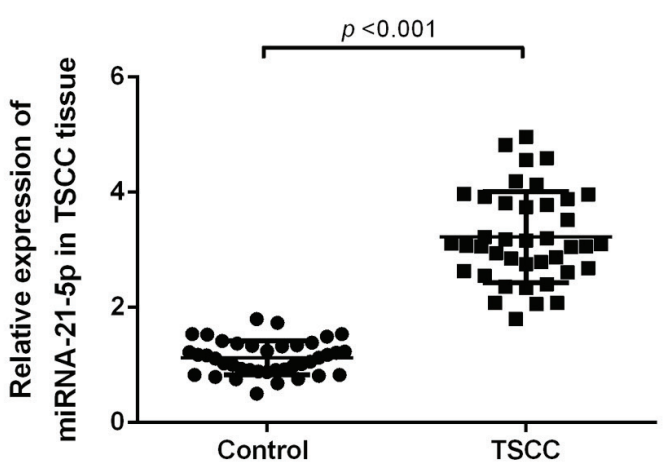

Figure 1. miR-21-5p is upregulated in TSCC tissue. The total RNA was extracted from the tongue tissues of 40 patients with TSCC, and diseased tissue was confirmed via H\&E staining. The expression of miR-21-5p was detected via reverse transcription-quantitative polymerase chain reaction analysis. TSCC histological classification was determined by H\&E staining. (A) Normal tongue tissue. (B) Poor differentiation. (C) Moderate differentiation. (D) Well-differentiated TSCC (magnification, x200). (E) miR-21-5p expression in TSCC tissues. Experiments were performed in triplicate. miR, microRNA; TSCC, tongue squamous cell carcinoma; H\&E, hematoxylin and eosin.

TCCA-3'; PDCD4 reverse, 5'-GATCCTAACTATGATGA-3'; GAPDH forward, 5'-TGTTGCCATCAATGACCCCTT-3'; GAPDH reverse, 5'-CTCCACGACGTACTCAGCG-3'. The expression levels of miRNA-21-5p were calculated using the $2^{-\Delta \Delta \mathrm{Cq}}$ method (10).

Transfection. miR-21-5p inhibitors were synthesized along with a corresponding negative control by Shanghai GenePharma Co., Ltd. Plasmid production and purification were performed by Shanghai GenePharma Co., Ltd. miR-21-5p inhibitor sequences (forward, 5'-UAGCUUAUC AGACUGAUGUUGA-3' and reverse, 5'-TCAACATCAGTC TGATAAGCTA-3') were cloned into the lentivirus without green fluorescence (Shanghai GeneChem Co., Ltd.). Polybrene (6 $\mu \mathrm{g} / \mathrm{ml}$; Shanghai GeneChem Co., Ltd.) and an appropriate dose of lentivirus $\left(1 \times 10^{8}\right)$ were added and incubated at $37^{\circ} \mathrm{C}$ for $24 \mathrm{~h}$. Cells transfected with lentivirus were screened with puromycin to increase transfection efficiency. The transfection efficiency was additionally identified via PCR.

Cell Counting Kit-8 (CCK-8). Next, 2,000 cells per well were seeded in the 96-well plate. Cell proliferation was detected using a CCK-8 assay (MedChemExpress) according to the manufacturer's protocol. The reproductive ability of cells was measured at $450 \mathrm{~mm}$ using a microplate reader.

Transwell assay. SCC-9 and Cal 27 cells were re-suspended without serum at a concentration of $1 \times 10^{5}$ cells $/ \mathrm{ml}$, and were then $(200 \mu \mathrm{l})$ seeded into the upper well of Matrigel-coated (Sigma Aldrich; Merck KGaA) $8 \mu \mathrm{m}$ pore Transwell inserts (Sigma Aldrich; Merck KGaA). DMEM (or RPMI-1640; Gibco; Thermo Fisher Scientific, Inc.) was added to the lower chamber containing $10 \%$ FBS. After $24 \mathrm{~h}$ of incubation at $37^{\circ} \mathrm{C}$, cells in the chamber were removed with a cotton swab.
Following fixed staining with $0.1 \%$ trypan blue for $20 \mathrm{~min}$ (Sigma Aldrich; Merck KGaA) at room temperature, images were captured at randomly selected fields and cells were counted under a light microscope (magnification, x200).

Western blot analysis. Total protein was extracted from the cells using radioimmunoprecipitation assay lysis buffer (Thermo Fisher Scientific, Inc.). A BCA protein assay kit was used to quantify the total protein. The proteins $(30 \mu \mathrm{g})$ were separated via $10 \%$ SDS-PAGE. The gels were transferred to polyvinylidene difluoride (PVDF) membranes, which were blocked with $5 \%$ skimmed milk at room temperature for $2 \mathrm{~h}$ and incubated with anti-Bax (1:1,000; cat. no. ab32503), anti-Bcl2 (1:1,000; cat. no. ab182858), anti-PI3K (1:1,000, ab127617), anti-AKT (1:1,000; cat. no. ab179463), anti-phosphorylated Forkhead Box O1 (p-FOXO1; 1:800; cat. no. ab52857) and anti-GAPDH (1:2,000; cat. no. ab181602) antibodies (all from Abcam) overnight at $4^{\circ} \mathrm{C}$. Following washing with PBS 3 times, the membranes were incubated with a secondary polyclonal peroxidase-labeled antibody (Goat Anti-Rabbit IgG; 1:4,000; Abcam; cat. no. ab205718) for $2 \mathrm{~h}$, and detected using enhanced chemiluminescence (Thermo Fisher Scientific, Inc.). Quantification of the bands was performed using ImageJ software (Version d1.47; National Institutes of Health).

Propidium iodide (PI) and Annexin V staining. SCC9 and Cal 27 cells were collected, washed and resuspended. Following the addition of $5 \mu \mathrm{l}$ Annexin $\mathrm{V}$ (BD Pharmingen; BD Biosciences) and $5 \mu \mathrm{l}$ PI (BD Pharmingen; BD Biosciences), cells were incubated at room temperature for $20 \mathrm{~min}$ in the dark, washed with PBS and re-suspended with $300 \mathrm{ml}$ of PBS. Cell apoptosis rate was calculated using Flow Jo software (v10.1.1 FlowJo LLC). 

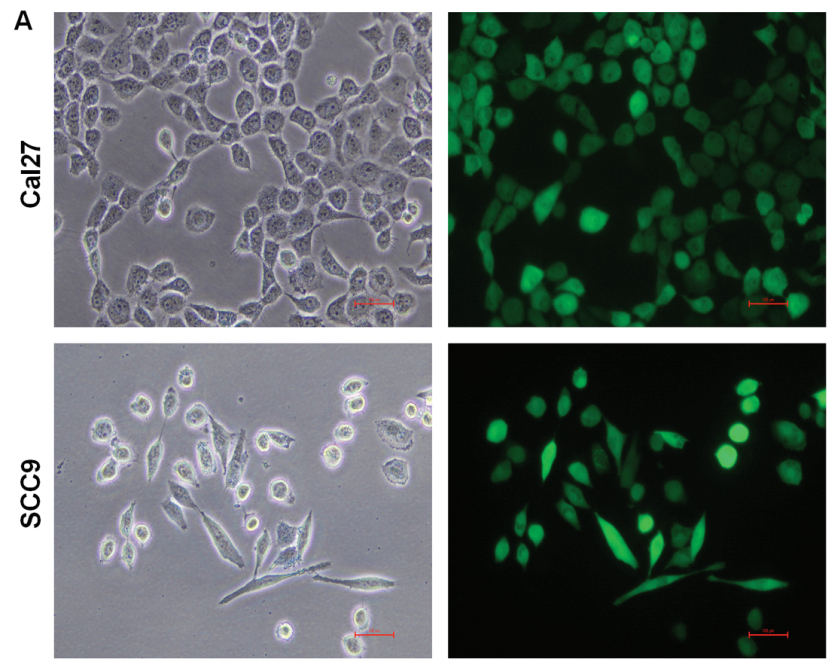

C

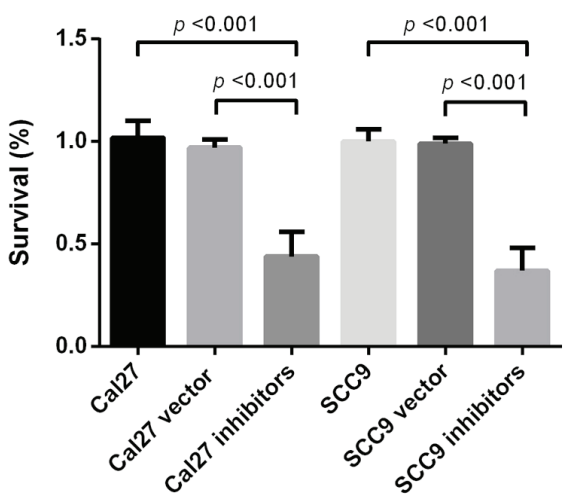

B

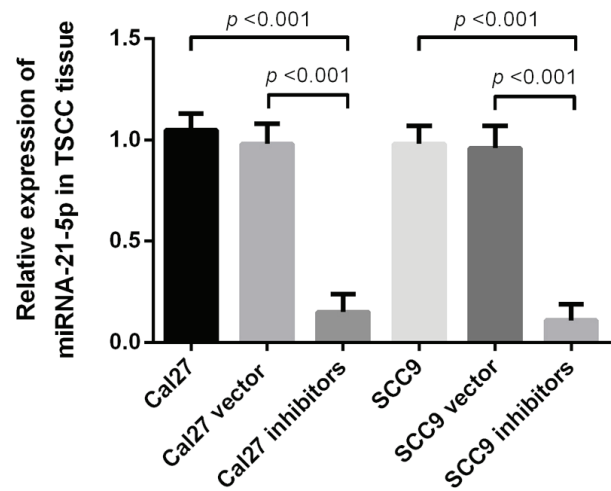

Figure 2. Effect of miR-21-5p on cell proliferation. In order to determine the function of miR-21-5p, miR-21-5p was transfected into SCC9 and Cal27 cells. The transfection efficiency of miR-21-5p was detected by RT-qPCR and the stability of the cells was detected by CCK-8 assay. (A) Transfection of miRNA-21-5p vectors in the TSCC cell lines. (B) Detection of miR-21-5p expression by RT-qPCR following transfection of the inhibitor. (C) CCK-8 assay detected cell proliferation in the TSCC cell lines. All experiments were performed in triplicate. miR, microRNA; RT-qPCR, reverse transcription-quantitative PCR; CCK8, Cell Counting Kit- 8 .

Target gene PDCD4 of miR-21-5p predicted by TargetScan. Targets of miR-21-5p were searched on TargetScan (www. targetscan.org/vert_71/) (11), and the results were further confirmed by PicTar29 (pictar.mdc-berlin.de/) and microRNA. org 30 (www.microrna.org) (12), suggesting PDCD4 is a potential target of miR-21-5p.

Luciferase reporter assay. The PDCD4 gene 3'-untranslated region (3'-UTR) sequence was cloned into a pMIR-REPORT vector (Ambion; Thermo Fisher Scientific, Inc.). Luciferase reporter plasmids of wild-type (WT)-PDCD4 mRNA and mutant $(\mathrm{Mu})$-PDCD4 mRNA were constructed using the clones. The cells were cultured for $24 \mathrm{~h}$, while the PDCD4-UTR-pMIR plasmid and miR-21-5p inhibitors or Mu-PDCD4-UTR-pMIR plasmid and miR-21-5p inhibitors were co-transfected into cells using Lipofectamine ${ }^{\mathrm{TM}} 2000$ transfection reagent (Thermo Fisher Scientific, Inc.) according to the manufacturer's protocol. The relative luciferase activity of PDCD4 was detected using a Dual-Luciferase Reporter assay after transfection for $48 \mathrm{~h}$. The Renilla luciferase values were normalized to that of firefly luciferase.

Statistical analysis. All data were presented as the mean \pm standard deviation. Statistical analysis was performed using GraphPad Prism (v6.0; GraphPad Software Inc.). The data were assessed using the unpaired two-tailed Student's t-test for comparisons between two groups, or one-way analysis of variance followed by Kruskal-Wallis test and Dunn's multiple comparison post-hoc test for comparisons between $>2$ groups. Correlations between the miR-21-5p and PDCD4 gene were evaluated using Spearman's coefficient of correlation. $\mathrm{P}<0.05$ was considered to indicate a statistically significant difference.

\section{Results}

Clinical characteristics of patients with TSCC. The clinical characteristics of the patients with TSCC are presented in Table I. According to the Tumor-Node-Metastasis classification for TSCC (Union for International Cancer Control, UICC 2010, 7th edition) (13), the patients were divided into 4 stages: Stage I $(n=4)$, stage II $(n=12)$, stage III $(n=17)$ and stage IV $(\mathrm{n}=7)$. Hematoxylin and eosin staining demonstrated that all patients had squamous cell carcinoma (Fig. 1A-D).

miR-21-5p is upregulated in TSCC tissues. miR-21-5p was detected in TSCC tissues via RT-qPCR. As presented in Fig. 1B, the expression levels of miR-21-5p were significantly 

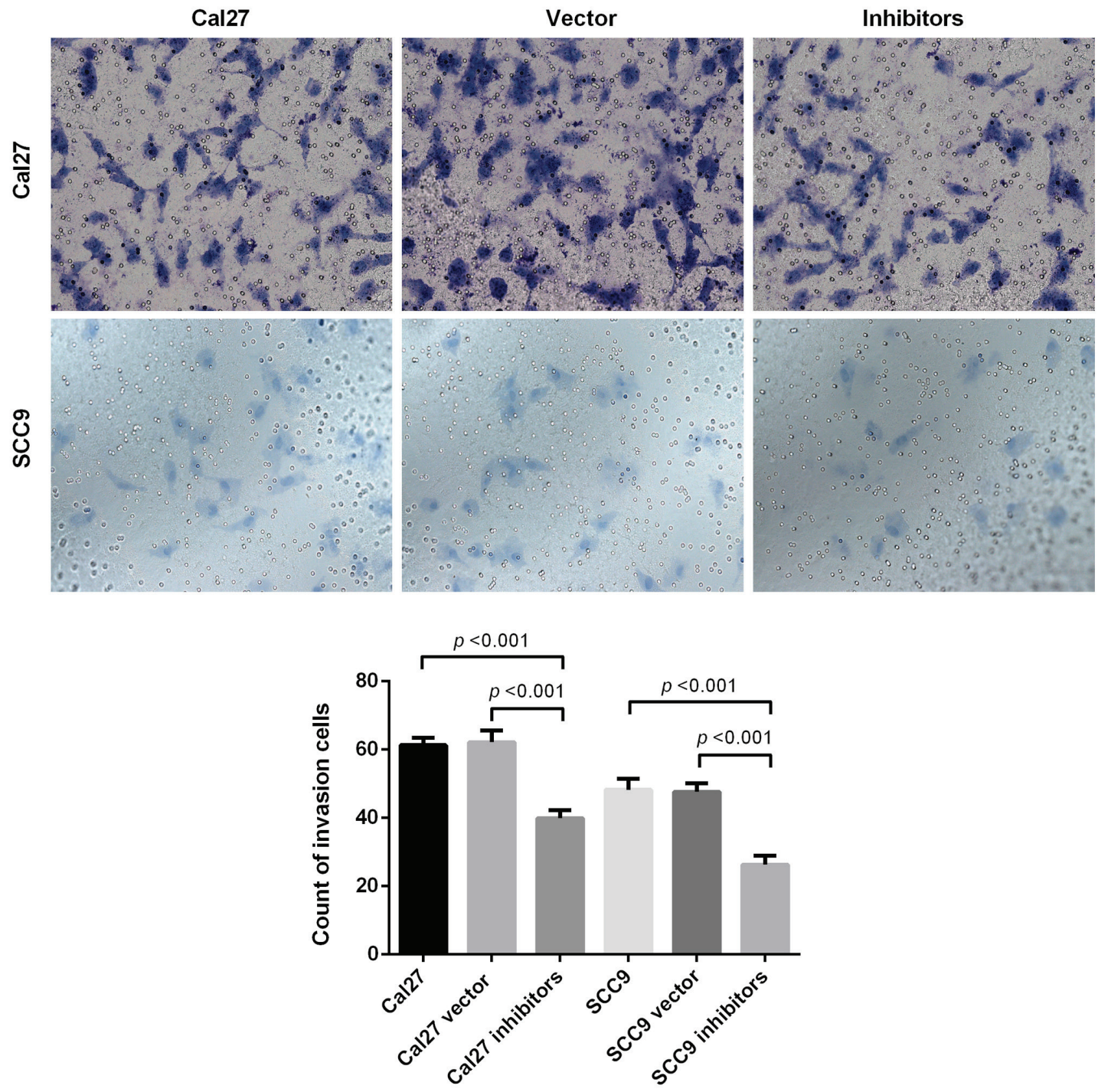

Figure 3. Analysis of the invasive capabilities of 2 cell lines regulated by miR-21-5p assessed with Transwell assays. Transwell assays were used to detect the invasive capabilities of SCC9 and Cal 27 cells following transfection with miR-21-5p inhibitor. Magnification, x200. miR, microRNA.

upregulated in TSCC tissue when compared with that of the control group (Fig. 1E).

Inhibition of miR-21-5p suppresses proliferation of Cal 27 cells and SCC 9 cells. To determine the function of miR-21-5p, miR-21-5p was knocked down in the Cal 27 and SCC9 cell lines. Following miR-21-5p knockdown, the expression levels of miR-21-5p in Cal 27 and SCC9 cells were significantly decreased when assessed via RT-qPCR (Fig. 2A and B). The CCK-8 assay subsequently determined the proliferation ability of Cal 27 and SCC9 cells following miR-21-5p knockdown. The results revealed that miR-21-5p knockdown markedly inhibited the proliferation levels of Cal 27 and SCC9 cells (Fig. 2C).

Inhibition of miR-21-5p suppresses the invasion ability of Cal 27 and SCC 9 cells. A Transwell assay was used to detect the invasive capabilities of Cal 27 and SCC9 cells. The results revealed that following miR-21-5p knockdown, the invasive capabilities of the Cal 27 and SCC9 cells decreased. The invasion assay demonstrated that the downregulation of miR-21-5p significantly inhibited the invasion capacity of Cal 27 and SCC9 cells (Fig. 3).

Inhibition of miR-21-5p promotes apoptosis of Cal 27 and SCC9 cells. To investigate the effect of miR-21-5p on Cal 27 and SCC9 cells, western blot analysis was used to detect apoptosis. The results revealed that the expression levels of the pro-apoptotic protein Bax increased significantly following miR-21-5p knockdown, while the expression level of anti-apoptosis protein $\mathrm{Bcl} 2$ was significantly decreased following knockdown of miR-21-5p. PI and Annexin V staining revealed that the early apoptosis of SCC 9 and Cal 27 cells significantly increased following miR-21-5p knockdown $(\mathrm{P}<0.05)$. These results demonstrate that the downregulation of miR-21-5p expression promotes the apoptosis of Cal 27 and SCC9 cells (Fig. 4).

PDCD4 is the target of miR-21-5p. miRNAs affect the expression levels of mRNA by binding to their 3'-UTR, and the role of miR-21-5p is closely associated with the function of the target gene [for example phosphatase and tensin 
A

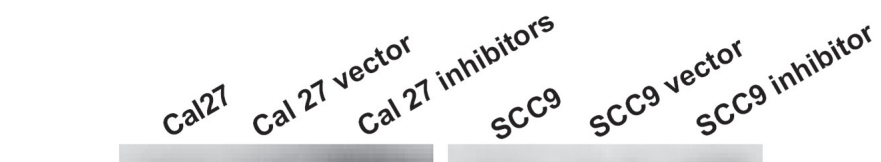

Bax
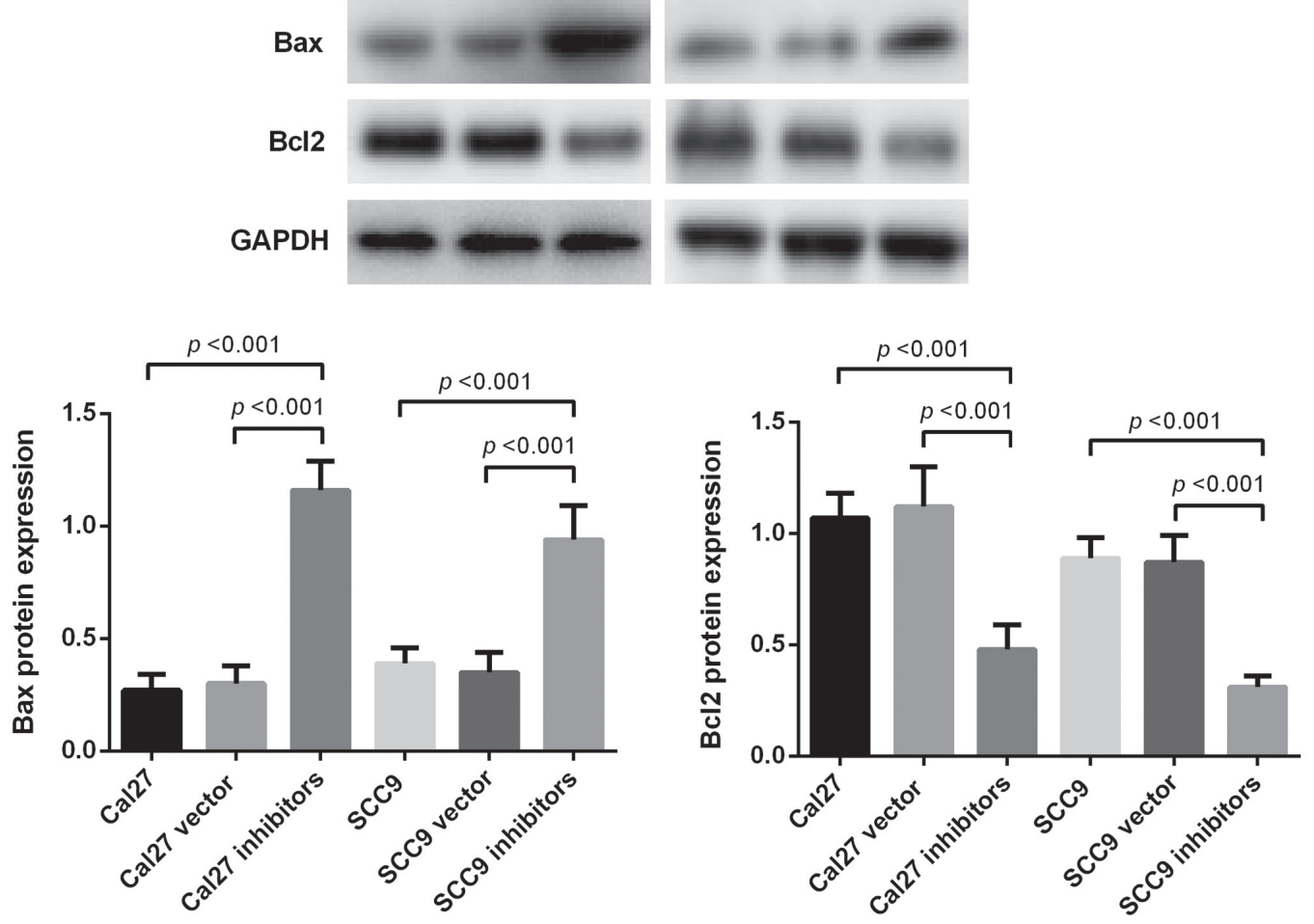

B
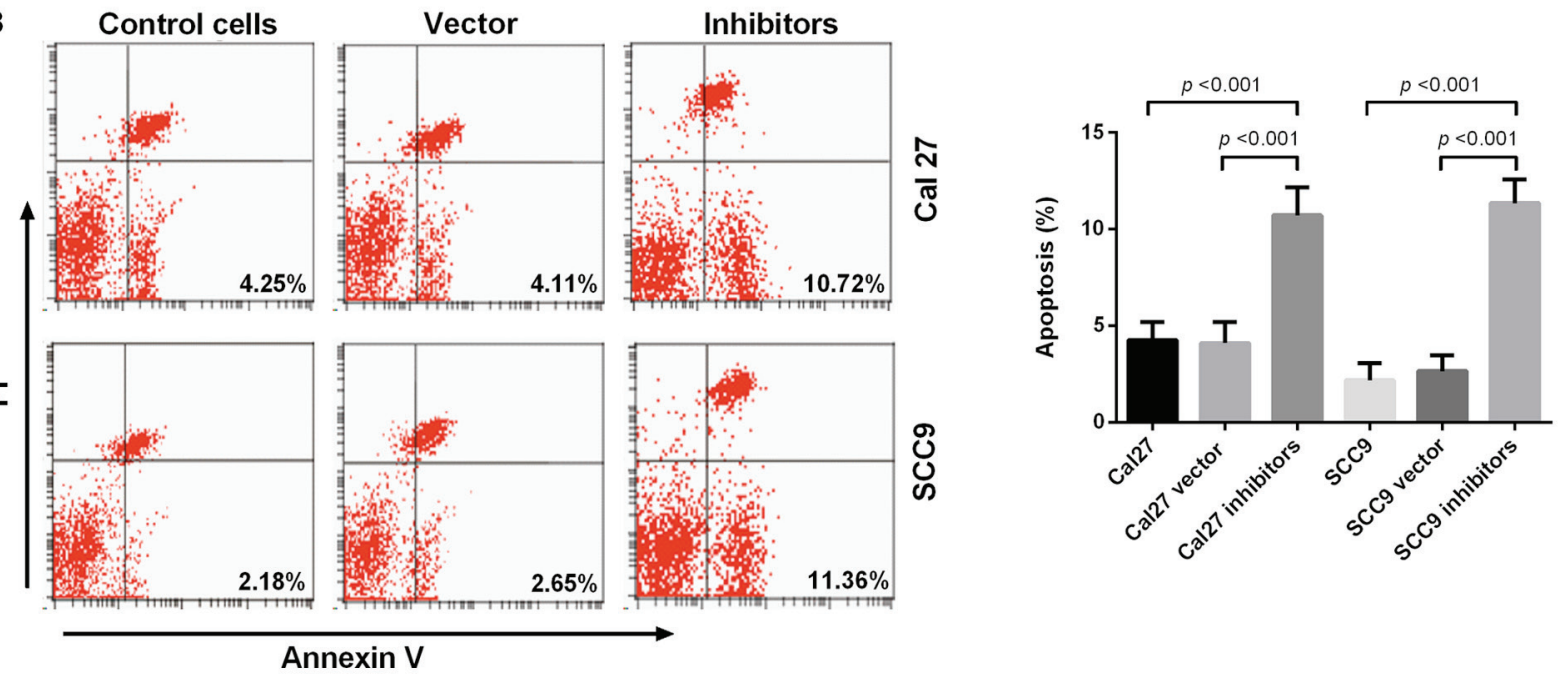

Figure 4. Inhibition of miR-21-5p promotes Cal 27 and SCC9 cells apoptosis. (A) The expression levels of apoptotic proteins Bax and Bcl2 were detected by western blot analysis. (B) Propidium iodide and Annexin V staining were used to detect the levels of early apoptosis of SCC9 and Cal 27 cells via flow cytometry. Inhibition of miR-21-5p expression significantly promoted apoptosis in the TSCC cell lines. All experiments were performed in triplicate. miR, microRNA; TSCC, tongue squamous cell carcinoma.

homolog (PTEN) and Smad7]. Target gene prediction indicated that PDCD4 may be a potential target of miR-21-5p (Fig. 5A). RT-qPCR was used to detect the expression levels of PDCD4 in TSCC tissues. The results revealed that the expression levels of PDCD4 decreased significantly in TSCC tissues (Fig. 5B), and the expression levels of miR-21-5p were negatively correlated with PDCD4 (Fig. 5C). To determine whether miRNA-21-5p directly targeted PDCD4, the present study cloned the 3'-UTR of PDCD4 downstream from the pMIR-CMV luciferase reporter. The results revealed that miRNA-21 decreased luciferase activity in WT-PDCD4-transfected cells (Fig. 5D). The luciferase assay demonstrated that PDCD4 was the target of miR-21-5p.

Apoptosis effect of miRNA-21-5p is determined via regulating the PI3K/Akt/FOXO1 signaling pathway. PI3K, AKT and p-FOXO1 protein expression levels were measured using western blot analysis (Fig. 6). The results revealed that the $\mathrm{PI} 3 \mathrm{~K}, \mathrm{AKT}$ and $\mathrm{p}-\mathrm{FOXO1}$ protein expression levels were significantly decreased following miR-21-5p knockdown both 
A

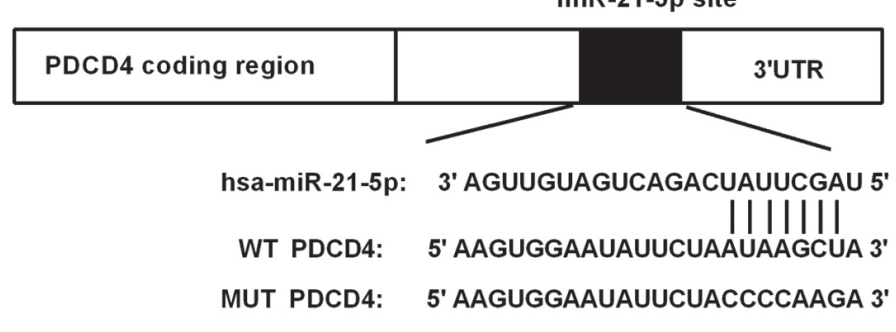

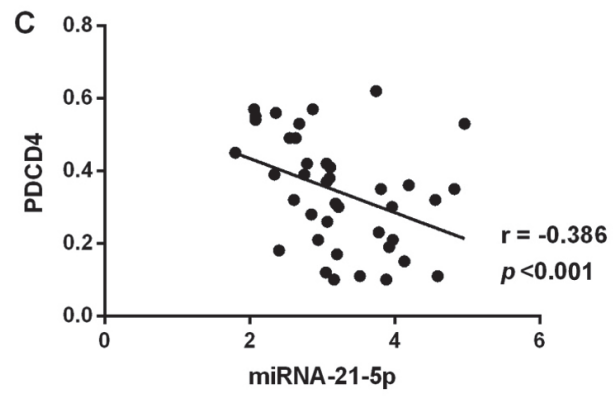
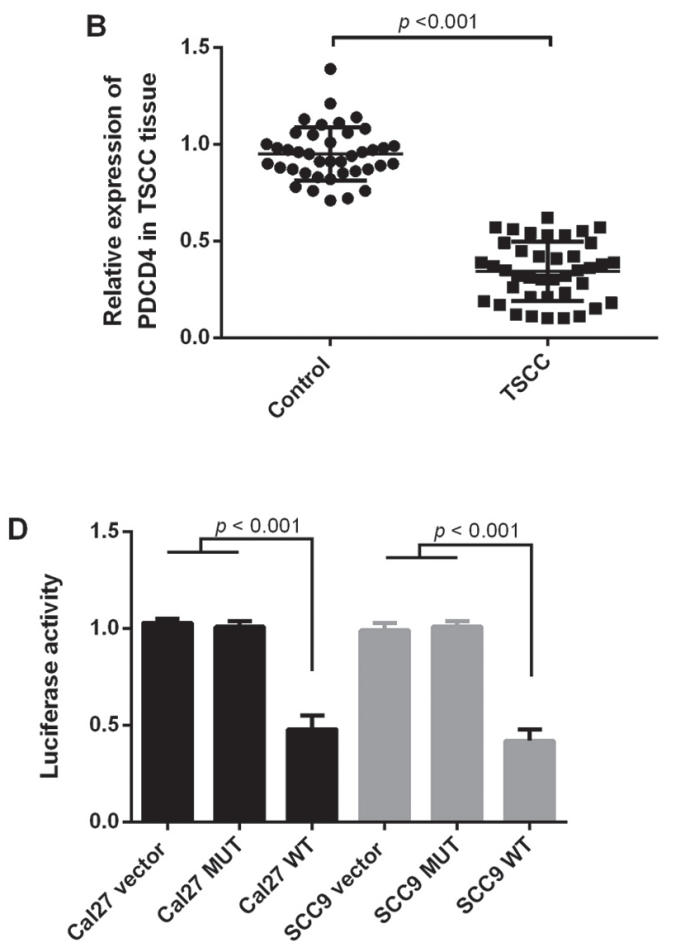

Figure 5. miR-21-5p promotes the apoptosis of the 2 TSCC cell lines by targeting PDCD4 following transfection of the inhibitor. (A) miR-21-5p binding with the 3'-UTR of PDCD4, as predicted online using TargetScan. PDCD4 was suggested to be the target of miR-21-5p. (B) PDCD4 gene expression was detected via reverse transcription-quantitative polymerase chain reaction, and it was identified that PDCD4 expression was significantly decreased in TSCC. (C) PDCD4 and miR-21-5p were demonstrated to be negatively correlated ( $r=-0.386 ; \mathrm{P}<0.001)$. (D) The 2 TSCC cell lines transfected with miR-21-5p inhibitors significantly suppressed the luciferase activity of WT-PDCD4 by luciferase reporter assay. miR, microRNA; PDCD4, programmed cell death 4; 3'-UTR, 3'-untranslated region; MUT, mutant; WT, wild-type.
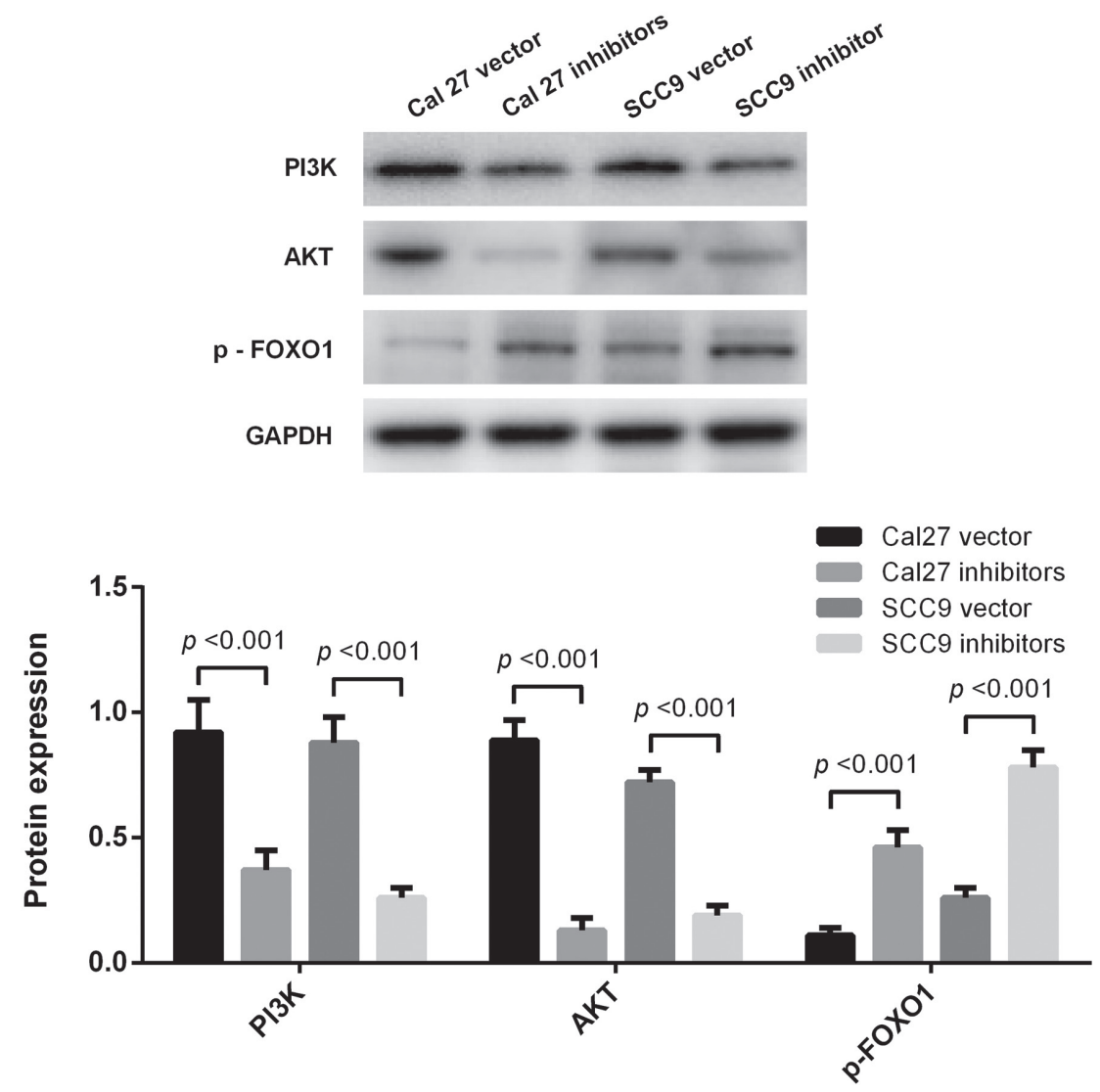

Figure 6. Apoptotic effect of miRNA-21-5p was determined via regulating PI3K/Akt/FOXO1 signaling. PI3K, AKT and p-FOXO1 protein expression levels were measured using western blot analysis. Inhibition of miRNA21-5p suppressed the PI3K/Akt/FOXO1 signaling pathway. Experiments were performed in triplicate. miRNA, microRNA; FOXO1, Forkhead Box O1; p-FOXO1, phosphorylated Forkhead Box O1. 
in Cal27 and SCC9 cells (Fig. 6). Inhibition of miR-21-5p promoted apoptosis of Cal27 and SCC9 cells via regulating the PI3K/AKT/FOXO1 signaling pathway.

\section{Discussion}

miRNAs are factors that regulates biological responses including apoptosis, proliferation, differentiation and invasion. miRNAs account for $\sim 1 \%$ of the genome, but they regulates $\sim 60 \%$ of gene activity (14). Under the catalysis of RNA polymerase II, miRNA is transformed into primitive miRNAs with a cap structure and polyA tail, then the primitive RNA forms a stem-loop structure, which is transferred from the nucleus to the cytoplasm with the help of transporter exportin-5. This stem-loop structure is cut to form mature miRNAs. Finally, this stem-loop structure is cleaved to form mature miRNAs (15). The mature miRNAs bind to the RNA-induced silencing complex (RISC) to form an asymmetric RISC complex, which binds to the 3'-UTR on the mRNA of the target gene to regulate the expression of that target gene (15).

miR-21-5p is a member of the miRNA family, which is highly expressed in the serum of patients with a number of different types of tumors and serves an anti-apoptotic role (9). miR-21-5p is closely associated with the molecular mechanisms underlying drug resistance, cell invasion and metastasis of tumors, among other processes (16-18). The results of the present study revealed that the expression levels of miRNA21-5p were upregulated in human TSCC, which was involved in the occurrence and development of tumors. CCK-8 and Transwell assay results demonstrated that the proliferative and invasive capabilities of the Cal 27 and SCC9 cell lines decreased following downregulation of miR-21-5p expression. Western blot analysis was used to detect the apoptotic and anti-apoptotic factors in the present study. The results revealed that downregulation of miR-21-5p promoted the apoptosis of Cal 27 and SC9 cells.

miR-21-5p directly or indirectly promotes the pathophysiological process of cells by regulating the expression of PDCD4, PTEN and tissue inhibitor of metalloproteinase-3 $(19,20)$. Usually one miRNA targets multiple genes. As PDCD4 has been frequently reported as a target of miR-21-5p, the role of miR-21-5p in PDCD4 expression in TSCC requires additional study (21). In the present study, the luciferase reporter assay revealed that PDCD4-UTR-pMIR (WT) was significantly increased by miR-21-5p inhibitors. This indicated that PDCD4 was the target of microRNA-21-5p, but its mechanism requires additional experimental confirmation.

$\mathrm{PI} 3 \mathrm{~K} / \mathrm{AKT} / \mathrm{FOXO1}$ is an important signaling pathway, which regulates progression in numerous different types of tumors. The PI3K/AKT pathway is closely associated with apoptosis. This pathway controls cell proliferation, growth, translation, migration and survival, and overactivation of this signaling pathway is associated with poor prognosis $(21,22)$. Activated PI3K may activate downstream protein kinase AKT; Activated AKT is able to phosphorylate Bax, inactivate Bax and inhibit Bax and Bcl-2 to form dimers, thereby resulting in Bcl-2 dissociation and an anti-apoptotic effect $(23,24)$. In the present study, the expression levels of PI3K/AKT signaling pathway-associated proteins were detected. It was demonstrated that inhibition of miR21-5p suppressed the PI3K/AKT/FOXO1 signaling pathway, suggesting that inhibiting the activation of the PI3K/AKT/FOXO1 signaling pathway may be a potential strategy for the treatment of TSCC.

In conclusion, downregulation of miR $21-5 p$ may effectively inhibit the proliferation and invasion of cancer cells and also promote the apoptosis of cancer cells. These data provide the basis for novel strategies for gene therapy of TSCC.

\section{Acknowledgements}

Not applicable.

\section{Funding}

The present study was funded by the Nature Science Foundation of Liaoning Province (grant no. 2015020326).

\section{Availability of data and materials}

The datasets used and/or analyzed during the present study are available from the corresponding author upon reasonable request.

\section{Authors' contributions}

$\mathrm{ZW}$ and $\mathrm{LT}$ conceived and designed the study. CL and ZT drafted the manuscript. Cell cultures were completed by ZT. The RT-qPCR protocol was performed by JT. Data analysis was performed by CL and ZX. All authors read and approved the final manuscript.

\section{Ethics approval and consent to participate}

The present study was approved by the Ethical Committees of Jinzhou Medical University on October 26, 2016 (approval no. JZH2016052). Informed consent was obtained from all patients included in the present study.

\section{Patient consent for publication}

Not applicable.

\section{Competing interests}

The authors declare that they have no competing interests.

\section{References}

1. Weatherspoon DJ, Chattopadhyay A, Boroumand S and Garcia I: Oral cavity and oropharyngeal cancer incidence trends and disparities in the United States: 2000-2010. Cancer Epidemiol 39: 497-504, 2015.

2. Ramqvist T, Grün N and Dalianis T: Human papillomavirus and tonsillar and base of tongue cancer. Viruses 7: 1332-1343, 2015.

3. Karatas OF, Oner M, Abay A and Diyapoglu A: MicroRNAs in human tongue squamous cell carcinoma: From pathogenesis to therapeutic implications. Oral Oncol 67: 124-130, 2017.

4. Sun L, Liang J, Wang Q, Li Z, Du Y and Xu X: MicroRNA-137 suppresses tongue squamous carcinoma cell proliferation, migration and invasion. Cell Prolif 49: 628-635, 2016.

5. Yuen PW, Lam KY, Chan AC, Wei WI and Lam LK: Clinicopathological analysis of local spread of carcinoma of the tongue. Am J Surg 175: 242-244, 1998. 
6. Rusthoven K, Ballonoff A, Raben D and Chen C: Poor prognosis in patients with stage I and II oral tongue squamous cell carcinoma. Cancer 112: 345-351, 2008

7. Chu H, Jia B, Qiu X, Pan J, Sun X, Wang Z and Zhao J: Investigation of proliferation and migration of tongue squamous cell carcinoma promoted by three chemokines, MIP-3 $\alpha$, MIP-1 $\beta$, and IP-10. Onco Targets Ther 10: 4193-4203, 2017.

8. Yan D, Cai X and Feng Y: miR-183 modulates cell apoptosis and proliferation in tongue squamous cell carcinoma SCC25 cell line. Oncol Res 24: 399-404, 2016.

9. Jiang Y, Zhang M, Guo T, Yang $\mathrm{C}$, Zhang $\mathrm{C}$ and Hao J: MicroRNA-21-5p promotes proliferation of gastric cancer cells through targeting SMAD7. Onco Targets Ther 11: 4901-4911, 2018.

10. Livak KJ and Schmittgen TD: Analysis of relative gene expression data using real-time quantitative PCR and the 2(-Delta Delta C(T)) method. Methods 25: 402-408, 2001.

11. Agarwal V, Bell GW, Nam JW and Bartel DP: Predicting effective microRNA target sites in mammalian mRNAs. eLife 4: e05005, 2015.

12. Betel D, Wilson M, Gabow A, Marks DS and Sander C: microRNA target predictions: The microRNA.org resource: Targets and expression. Nucleic Acids Res 36: D149-D153, 2008

13. Sobin L, Gospodarowicz M and Wittekind C (eds): UICC International Union Against Cancer TNM Classification of Malignant Tumors. 7th edition. West Sussex, Wiley-Blackwell, United Kingdom, 2009.

14. Fang H, Xie J, Zhang M, Zhao Z, Wan Y and Yao Y: miRNA-21 promotes proliferation and invasion of triple-negative breast cancer cells through targeting PTEN. Am J Transl Res 9: 953-961, 2017.

15. Zhang Z, Li Z, Gao C, Chen P, Chen J, Liu W, Xiao S and Lu H: miR-21 plays a pivotal role in gastric cancer pathogenesis and progression. Lab Invest 88: 1358-1366, 2008.

16. Mohr AM and Mott JL: Overview of microRNA biology. Semin Liver Dis 35: 3-11, 2015.
17. Ricci C, Marzocchi C and Battistini S: MicroRNAs as biomarkers in amyotrophic lateral sclerosis. Cells 7: pii: E219, 2018.

18. Bourguignon LY: Matrix hyaluronan promotes specific MicroRNA upregulation leading to drug resistance and tumor progression. Int J Mol Sci 17: 517, 2016.

19. Zhao Y, Zhao L, Ischenko I, Bao Q, Schwarz B, Nieß H, Wang Y, Renner A, Mysliwietz J, Jauch KW, et al: Antisense inhibition of microRNA-21 and microRNA-221 in tumor-initiating stem-like cells modulates tumorigenesis, metastasis, and chemotherapy resistance in pancreatic cancer. Target Oncol 10: 535-548, 2015.

20. Liu ZL, Wang H, Liu J and Wang ZX: MicroRNA-21 (miR-21) expression promotes growth, metastasis, and chemo- or radioresistance in non-small cell lung cancer cells by targeting PTEN. Mol Cell Biochem 372: 35-45, 2013.

21. Wang Z, Yao W, Li K, Zheng N, Zheng C, Zhao X and Zheng S: Reduction of miR-21 induces SK-N-SH cell apoptosis and inhibits proliferation via PTEN/PDCD4. Oncol Lett 13: 4727-4733, 2017.

22. Pardini B, De Maria D, Francavilla A, Di Gaetano C, Ronco G and Naccarati A: MicroRNAs as markers of progression in cervical cancer: A systematic review. BMC Cancer 18: 696, 2018.

23. Syed DN, Afaq F, Sarfaraz S, Khan N, Kedlaya R, Setaluri V and Mukhtar H: Delphinidin inhibits cell proliferation and invasion via modulation of Met receptor phosphorylation. Toxicol Appl Pharmacol 231: 52-60, 2008.

24. Hao Y, Huang J, Ma Y, Chen W, Fan Q, Sun X, Shao M and Cai $\mathrm{H}$ : Asiatic acid inhibits proliferation, migration and induces apoptosis by regulating Pdcd4 via the PI3K/Akt/mTOR/p70S6K signaling pathway in human colon carcinoma cells. Oncol Lett 15: 8223-8230, 2018.

This work is licensed under a Creative Commons Attribution-NonCommercial-NoDerivatives 4.0 International (CC BY-NC-ND 4.0) License. 